\title{
Effective Interaction Potentials in the Uppermost Landau Level
}

\author{
O. Ciftja $\cdot$ J. Quintanilla
}

Received: 22 July 2009 / Accepted: 1 December 2009 / Published online: 31 December 2009

(C) Springer Science+Business Media, LLC 2009

\begin{abstract}
We consider a quantum Hall system of electrons confined to the uppermost Landau level and assume that the lower Landau levels are full and inert causing no Landau level mixing. While it is known that the problem of electrons interacting with the Coulomb interaction in a higher Landau level is mathematically equivalent to the problem of electrons in the lowest Landau level interacting with an effective interaction, the way the effective interaction can be calculated is not unique. We focus on the details of two different calculations of such effective interaction potentials in the uppermost Landau level and discuss the influence of one or another form of the effective potential on the stability of various correlated electronic phases in the quantum Hall regime.
\end{abstract}

Keywords Quantum Hall systems · Landau levels

\section{Introduction}

The phase diagram of a two-dimensional electron gas (2DEG) in weak magnetic field becomes quite interesting when high Landau level-s (LL-s) with indices $n \geq 2$ are partially filled. Transport experiments in very high mobility $\mathrm{GaAs} / \mathrm{Al}_{x} \mathrm{Ga}_{1-x} \mathrm{As}$ heterostructures [1] have established the existence of highly anisotropic electronic states around even-denominator filling factors $f=9 / 2,11 / 2$, etc., states that were likely expected to be isotropic Fermi liquids. Earlier theoretical work [2] explained anisotropy in terms of a striped charge density wave (CDW) that stabilizes in weak

O. Ciftja (凶)

Department of Physics, Prairie View A\&M University, Prairie View, TX 77446, USA

e-mail: ogciftja@pvamu.edu

J. Quintanilla

ISIS Facility STFC Rutherford Appleton Laboratory, Harwell Science and Innovation Campus,

Didcot, OX11 0QX, UK 
magnetic fields. While a stripe CDW phase is sufficient to explain anisotropy, other structures may lead to an anisotropic response as well [3]. A mechanism in which a compressible Fermi liquid state with circular Fermi surface spontaneously becomes anisotropic in presence of interactions is through the Pomeranchuk instability (PI) effect originally studied in the context of Landau theory of 3D Fermi liquid systems [4]. An anisotropic Fermi liquid state can be most easily pictured as a state with a deformed Fermi surface, for example, 2D plane-waves, $\left\{\varphi_{\mathbf{k}}\left(\mathbf{r}_{i}\right)\right\}$ that fill an elliptical Fermi sea [5]. Another possible scenario is to have anisotropy emerge at the two-particle and not single-particle level, an idea incorporated in the broken rotational symmetry (BRS) wave function $[6,7]$.

Studies of 3D Fermi systems [8] as well as more recent work in 2D Fermi systems [9] has indicated that the occurrence of the PI effect crucially depends on the form of the (effective) interaction potential between fermions. These studies suggest that a PI transition may occur only if the (effective) interaction potential between the fermions has non-monotonic features at some characteristic length scale. In this work, we consider a quantum Hall system of electrons confined to the uppermost LL and calculate the effective interaction potential between electrons using two slightly different approaches. We discuss the impact of one or another form of the effective potential on the stability of various correlated electronic phases that may arise at or in vicinity of the half-filled states in high LL-s.

\section{The "Frozen Out" Interaction Potential}

We consider a quantum Hall state in which the $n$-th LL is partially occupied. Because the lower LL-s are completely filled, we may ignore them, thus adopt the "frozen LL" approximation [10]. For the purpose of calculations this implies that the quantum Hall state under consideration has a filling factor, $f_{n}$ where $f_{n}$ is the partial filling factor of the $n$-th LL. Within this model, the corresponding density of the system should be taken as $\rho_{n}=f_{n} /\left(2 \pi l_{0}^{2}\right)$ even though the real filling factor is $f=2 n+f_{n}\left(l_{0}\right.$ is the magnetic length). We want to prove that the problem of particles interacting with an arbitrary interaction, $v\left(\left|\mathbf{r}_{1}-\mathbf{r}_{2}\right|\right)$ in a high LL is mathematically equivalent to the problem of particles in the lowest LL interacting with an effective interaction, $v_{n}\left(\left|\mathbf{r}_{1}-\mathbf{r}_{2}\right|\right)$ defined by:

$$
\left\langle n, m_{1}^{\prime} ; n, m_{2}^{\prime}\left|v\left(\left|\mathbf{r}_{1}-\mathbf{r}_{2}\right|\right)\right| n, m_{1} ; n, m_{2}\right\rangle=\left\langle 0, m_{1}^{\prime} ; 0, m_{2}^{\prime}\left|v_{n}\left(\left|\mathbf{r}_{1}-\mathbf{r}_{2}\right|\right)\right| 0, m_{1} ; 0, m_{2}\right\rangle,
$$

where $n$ is the LL index and $m_{i}$-s are angular momentum quantum numbers. One solves this problem by first writing the interaction potential in standard 2D Fourier space and then using the well-known formula: $\left\langle n, m^{\prime}\left|e^{ \pm i \mathbf{k r}}\right| n, m\right\rangle=$ $L_{n}\left(\frac{|\mathbf{k}|^{2} l_{0}^{2}}{2}\right)\left\langle 0, m^{\prime}\left|e^{ \pm i \mathbf{k} \mathbf{r}}\right| 0, m\right\rangle$, where $L_{n}(x)$ are Laguerre polynomials. Assuming a Coulomb interaction potential, $v\left(\left|\mathbf{r}_{1}-\mathbf{r}_{2}\right|\right)=e^{2} /\left(\epsilon\left|\mathbf{r}_{1}-\mathbf{r}_{2}\right|\right)$, one proceeds to perform an inverse 2D Fourier transform to find:

$$
v_{n}^{A}(r)=\frac{e^{2}}{\epsilon l_{0}} \int_{0}^{\infty} d q J_{0}(q r)\left[L_{n}\left(\frac{q^{2}}{2}\right)\right]^{2},
$$


where $r=\left|\mathbf{r}_{1}-\mathbf{r}_{2}\right| / l_{0}$ is a dimensionless separation distance, $q=|\mathbf{k}| l_{0}$, and $J_{0}(x)$ is a 0 -th order Bessel function.

\section{The "Projected" Interaction Potential}

The interaction potential, $\hat{V}_{n}$ of electrons confined to the partially filled $n$-th LL can be written as:

$$
\hat{V}_{n}=\frac{1}{2} \int d^{2} r_{1} \int d^{2} r_{2} \Psi_{n}^{\dagger}\left(\mathbf{r}_{1}\right) \Psi_{n}\left(\mathbf{r}_{1}\right) v\left(\left|\mathbf{r}_{1}-\mathbf{r}_{2}\right|\right) \Psi_{n}^{\dagger}\left(\mathbf{r}_{2}\right) \Psi_{n}\left(\mathbf{r}_{2}\right),
$$

where the field operators consist of states in the $n$-th LL: $\Psi_{n}(\mathbf{r})=\sum_{m}\langle\mathbf{r} \mid n, m\rangle \hat{a}_{n, m}$ and $\Psi_{n}^{\dagger}(\mathbf{r})=\sum_{m}\langle n, m \mid \mathbf{r}\rangle \hat{a}_{n, m}^{\dagger}$. One starts with the electronic density in $n$-th LL, $\rho_{n}(\mathbf{r})=\Psi_{n}^{\dagger}(\mathbf{r}) \Psi_{n}(\mathbf{r})$ which can be written in 2D Fourier space as $\rho_{n}(\mathbf{k})=$ $\int d^{2} r e^{-i \mathbf{k r}} \rho_{n}(\mathbf{r})=F_{n}(k) \bar{\rho}(\mathbf{k})$ where $\bar{\rho}(\mathbf{k})$ is the projected density operator in the $n$-th LL and $F_{n}(k)$ is a form factor given by $F_{n}(k)=L_{n}\left(\frac{k^{2} l_{0}^{2}}{2}\right) \exp \left(-\frac{k^{2} l_{0}^{2}}{4}\right)$. One ends up with a $n$-th LL projected interaction potential of the form $\hat{V}_{n}=$ $\frac{1}{2} \sum_{\mathbf{k}} v(k) \rho_{n}(\mathbf{k}) \rho_{n}(-\mathbf{k})=\frac{1}{2} \sum_{\mathbf{k}} v_{n}(k) \bar{\rho}(\mathbf{k}) \bar{\rho}(-\mathbf{k})$ where $v_{n}(k)=v(k)\left[F_{n}(k)\right]^{2}$ represents an effective interaction potential among electrons [11, 12]. An inverse 2D Fourier transform of $v_{n}(k)$ results in

$$
v_{n}^{B}(r)=\frac{e^{2}}{\epsilon l_{0}} \int_{0}^{\infty} d q J_{0}(q r)\left[L_{n}\left(\frac{q^{2}}{2}\right)\right]^{2} \exp \left(-\frac{q^{2}}{2}\right) .
$$

\section{Conclusions}

Plots of two interaction potentials which, to avoid confusion, were labeled $v_{n}^{A}(r)$ and $v_{n}^{B}(r)$ are shown in Fig. 1 for $n=0,1$ and 2. The first interaction potential, $v_{n}^{A}(r)$ is monotonic and mimics the bare Coulomb potential with the only difference that it becomes more "hard-core" as $n$ increases. Given the absence of any sharp
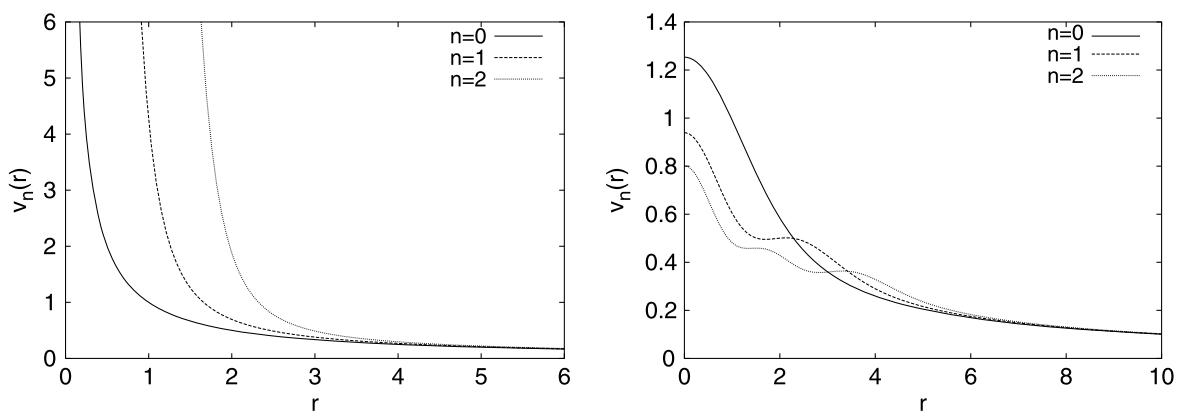

Fig. 1 Plot of $v_{n}^{A}(r)$ (left) and $v_{n}^{B}(r)$ (right) in units of $e^{2} /\left(\epsilon l_{0}\right)$ as a function of the dimensionless distance $r=r_{12} / l_{0}$ 
features in this potential, such an interaction should not lead to a PI transition in the half-filled states in high LL-s [8]. Quite differently, the non-monotonicity of the interaction potential $v_{n}^{B}(r)$ suits a PI transition of Fermi liquid states, at least at filling factors $2 n+1 / 2$ or $2 n+3 / 2(n=2,3, \ldots)$, where a Fermi surface is expected to exist. However, as previously noted [13], anisotropy similar to a PI deformed Fermi liquid state can also be manifested by a BRS phase. In earlier studies at filling factors $f=2 n+1 / 2$, a BRS liquid crystalline wave function

$$
\Psi_{\alpha}=\hat{P}_{n} \prod_{j<k}^{N}\left(z_{j}-z_{k}+\alpha\right)\left(z_{j}-z_{k}-\alpha\right) \times \exp \left(-\sum_{k=1}^{N} \frac{\left|z_{k}\right|^{2}}{4 l_{0}^{2}}\right) \operatorname{det}\left[\varphi_{\mathbf{k}}\left(\mathbf{r}_{i}\right)\right]
$$

has been used to calculate the energy competition between BRS and isotropic phases, though only with the monotonic potential $v_{n}^{A}(r)$ as choice [6]. While anisotropic states are more pronounced at $9 / 2$ and $11 / 2(n=2)$ it is known that anisotropy remains sizeable in a range of filling factors, $f=2 n+1 / 2 \pm \Delta f_{n}$ where $\Delta f_{n} \approx$ $0.2(n=2)$. Electronic states away from $2 n+1 / 2$ are not supposed to be Fermi liquid states. Thus, it is unlikely that anisotropy in this regime originates from the PI mechanism. In such a case, a better candidate to compete with the stripe CDW state might be a BRS liquid crystalline state. Even in the case of a monotonic potential such as $v_{n}^{A}(r)$, it has been found that for $f=2 n+1 / m$ ( $m$-odd), a BRS anisotropic state for $n=2$ has a lower energy than its isotropic counterpart [14, 15]. A non-monotonic potential like $v_{n}^{B}(r)$ should further enhance a BRS liquid crystalline phase that we intend to study through quantum Monte Carlo methods [16].

Acknowledgements O. C. acknowledges financial support from the National Science Foundation Grant No. DMR-0804568. J.Q. acknowledges financial support from CCLRC (now STFC) in association with St. Catherine's College, Oxford.

\section{References}

1. M.P. Lilly, K.B. Cooper, J.P. Eisenstein, L.N. Pfeiffer, K.W. West, Phys. Rev. Lett. 82, 394 (1999)

2. R. Moessner, J.T. Chalker, Phys. Rev. B 54, 5006 (1996)

3. E. Fradkin, S.A. Kivelson, Phys. Rev. B 59, 8065 (1999)

4. I.Ia. Pomeranchuk, J. Exp. Theor. Phys. 35, 524 (1958)

5. Q.M. Doan, E. Manousakis, Phys. Rev. B 75, 195433 (2007)

6. O. Ciftja, C. Wexler, Phys. Rev. B 65, 205307 (2002)

7. C. Wexler, O. Ciftja, Int. J. Mod. Phys. B 20, 747 (2006)

8. J. Quintanilla, A.J. Schofield, Phys. Rev. B 74, 115126 (2006)

9. J. Quintanilla, M. Haque, A.J. Schofield, Phys. Rev. B 78, 035131 (2008)

10. A.H. MacDonald, S.M. Girvin, Phys. Rev. B 33, 4009 (1986)

11. G. Murthy, R. Shankar, Rev. Mod. Phys. 75, 1101 (2003)

12. M.O. Goerbig, P. Lederer, C. Morais Smith, Phys. Rev. B 69, 115327 (2004)

13. O. Ciftja, C. Wexler, Physica B 403, 1511 (2008)

14. O. Ciftja, C.M. Lapilli, C. Wexler, Phys. Rev. B 69, 125320 (2004)

15. C. Wexler, O. Ciftja, J. Phys., Condens. Matter 14, 3705 (2002)

16. O. Ciftja, Europhys. Lett. 74, 486 (2006) 